\title{
False-Negative Nasopharyngeal Swab RT-PCR Assays in Typical COVID-19: Role of Ultra-low-dose Chest CT and Bronchoscopy in Diagnosis
}

\author{
Marco Marando ${ }^{1}$, Adriana Tamburello $^{1}$, Pietro Gianella ${ }^{1,2}$ \\ ${ }^{1}$ General Internal Medicine, Ente Ospedaliero Cantonale, Lugano, Switzerland \\ 2 Pneumology, Ente Ospedaliero Cantonale, Lugano, Switzerland
}

Received: 23/04/2020

Accepted: 23/04/2020

Published: 24/04/2020

\begin{abstract}
How to cite this article: Marando M, Tamburello A, Gianella P. False-negative nasopharyngeal swab RT-PCR assays in typical COVID-19: role of ultra-low-
\end{abstract} dose chest CT and bronchoscopy diagnosis. EJCRIM 2020;7: doi:10.12890/2020_001680.

Conflicts of Interests: The Authors declare that there are no competing interests.

This article is licensed under a Commons Attribution Non-Commercial 4.0 License

\section{ABSTRACT}

On 11 March 2020, the WHO declared COVID-19 a pandemic and global health emergency. We describe the clinical features and role of ultra-low-dose chest computed tomography (CT) and bronchoscopy in the diagnosis of coronavirus disease (COVID-19). In our patient, who was highly suggestive clinically and radiologically for COVID-19, we had two false-negative results for nasopharyngeal and oral swab reverse-transcriptase polymerase chain reaction (RT-PCR) assays for severe acute respiratory syndrome coronavirus 2 (SARS-CoV-2). Eventually, we confirmed the diagnosis using bronchoscopy and bronchoalveolar lavage (BAL).

\section{LEARNING POINTS}

- Clinical and laboratory findings in COVID-19 are unspecific.

- Chest CT has a diagnostic sensitivity comparable to nasopharyngeal swab RT-PCR assay but lacks specificity.

- RT-PCR assays on biological specimens, particularly nasopharyngeal swabs, are considered the diagnostic gold standard.

- Bronchoscopy and bronchoalveolar lavage can help confirm the diagnosis and should be performed in patients in whom diagnosticdriven treatment for COVID-19, such as tocilizumab or remdesivir, is being considered.

\section{KEYWORDS}

Coronavirus, COVID-19, RT-PCR, ultra-low-dose chest CT, bronchoscopy

\section{INTRODUCTION}

In December 2019, several pneumonia cases of unknown aetiology were reported in Wuhan, Hubei, China. The disease was soon identified as being caused by a novel coronavirus initially called 2019 novel coronavirus (2019-nCoV) and subsequently officially named SARS-CoV-2 by the WHO on 11 February $2020^{[1,2]}$.

As of 21 April 2020, there were 2,402,250 laboratory-confirmed cases and 163,097 confirmed deaths in 213 countries, areas or territories. Switzerland had 27,862 cases and 1141 confirmed deaths ${ }^{[3]}$. RT-PCR assays for SARS-CoV-2 on biological specimens, particularly nasopharyngeal swabs, are regarded as the diagnostic gold standard.

\section{CASE DESCRIPTION}

On 11 March 2020, a 57-year-old Caucasian woman was referred to our emergency department because of a 2-day history of dyspnoea at rest and a 6-day history of fever up to $39^{\circ} \mathrm{C}$. She had been treated by her general practitioner since 7 March for fever interpreted as a urinary tract infection due to Escherichia coli. In addition to fever, she also had constitutional symptoms such as myalgia and a mild tension 
headache. Her medical history was otherwise significant for obesity (BMI $39.4 \mathrm{~kg} / \mathrm{m}^{2}$ ), dyslipidaemia, duplex kidney (duplicated collecting system) with recurring nephrolithiasis and fibromyalgia. She was being treated with duloxetine and pregabalin. She had no food, pollen or drug allergies. She had never smoked tobacco, and did not use alcohol or illicit drugs. She denied other symptoms. She denied having left the country or, specifically, having gone to Italy or China in the last 14 days or to have been exposed to a patient affected by COVID-19. On physical examination, the patient appeared dyspnoeic. Vital signs were significant for a body temperature of $38.8^{\circ} \mathrm{C}$, oxygen saturation of $90 \%$ on ambient air, and a respiratory rate of $16 / \mathrm{min}$. Lung sounds were decreased at bases, with right lower-lobe rales. Chest radiography showed bilateral opacities with peripheral distribution, highly suggestive for interstitial pneumonia (Fig. 1). The patient's main characteristics are shown in Fig. 2. The ECG showed a normal sinus rhythm. A rapid test for influenza A, B and respiratory syncytial virus was negative. Urinalysis was unremarkable. Urinary antigen tests for Streptococcus pneumoniae and serotype 1 Legionella pneumophila were negative. Empiric antibiotic therapy with ceftriaxone was started.

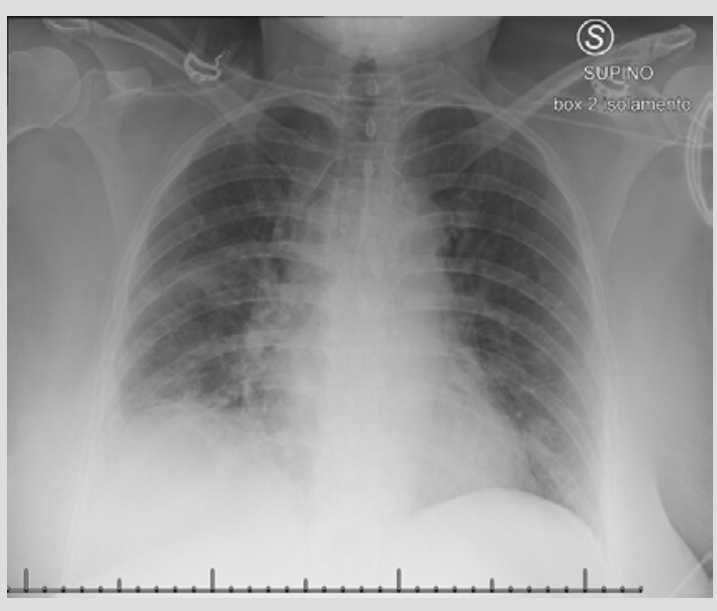

Figure 1. Admission chest $x$-ray shows increased and thickened right lower lung markings, suggesting bronchitis and interstitial pneumonia

Figure 2. Patient characteristics during hospitalisation

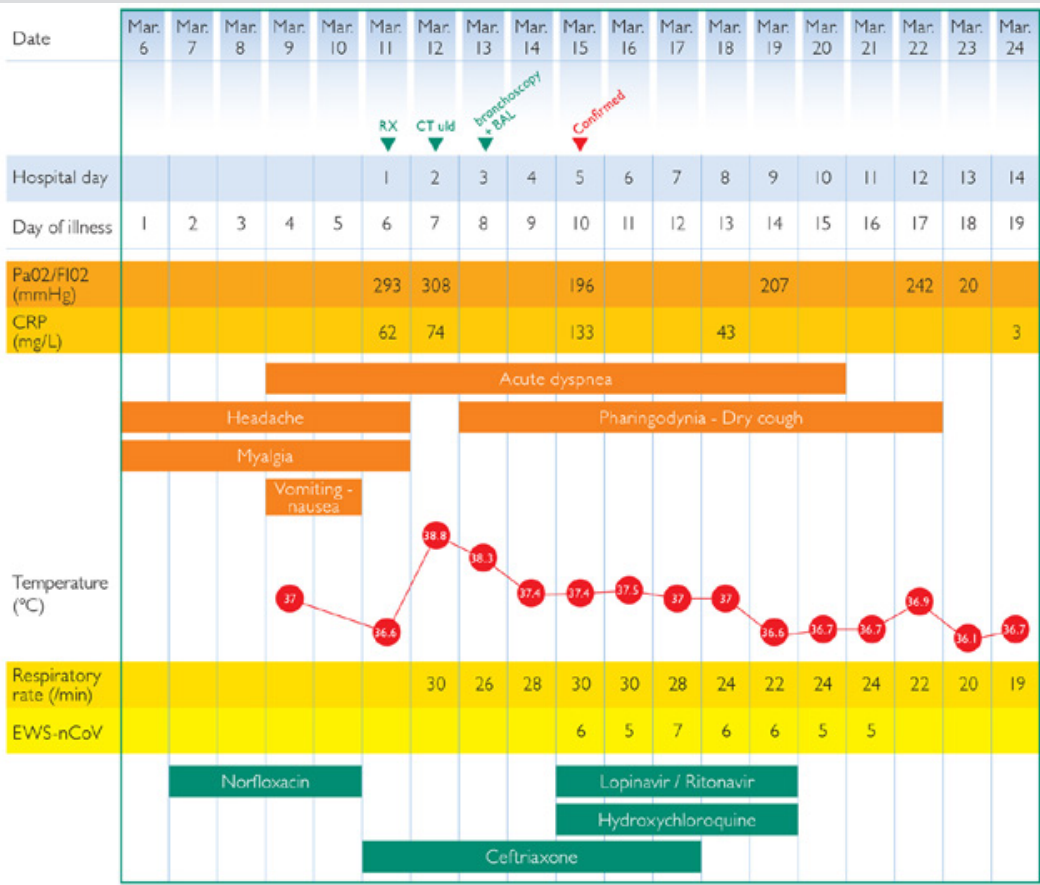

Given the outbreak of SARS-CoV-2 in Switzerland and nearby Italy, we obtained a nasopharyngeal and oral swab, which was reported negative for SARS-CoV-2 within 12 hours. The nasopharyngeal swab was taken by a doctor, supervised by a colleague, according to WHO guidelines. A nasopharyngeal swab RT-PCR assay for viral respiratory pathogens was reported back within 48 hours as negative for all pathogens tested, including influenza A and B, parainfluenza, respiratory syncytial virus, rhinovirus, adenovirus, and four common coronavirus strains known to cause illness in humans (HKU1, NL63, 229E and OC43). Peripheral blood cultures did not show any growth. Because the clinical and radiological picture was highly suspicious for COVID-19, on 13 March we performed an ultra-low-dose chest CT (Fig. 3), which showed bilateral multifocal ground-glass opacities, predominant in the inferior lobes, with confluent lesions. We repeated the nasopharyngeal and oral swabs for SARS-CoV-2 and HIV, which were reported negative within 12 hours. On 14 March, we performed bed-side fibre-optic bronchoscopy through a laryngeal mask airway, under the supervision of an anaesthetist who provided sedation with propofol and as-needed ventilation with a bag valve mask. We instilled $100 \mathrm{ml}$ sterile $\mathrm{NaCl} 0.9 \%$ into the middle lobe. The BAL was screened for cytology, bacteria, mycobacteria, fungi and SARS-CoV-2. Within 48 hours it was reported positive for SARS-CoV-2. Cellular differentiation in BAL showed macrophages $83.3 \%$, lymphocytes $11.5 \%$, neutrophils $5.2 \%$ and eosinophils $0 \%$. Therapy with lopinavir/ ritonavir and hydroxychloroquine was prescribed for 5 days. By 18 March, the patient's clinical status had improved and she was discharged from hospital on 25 March 2020. 

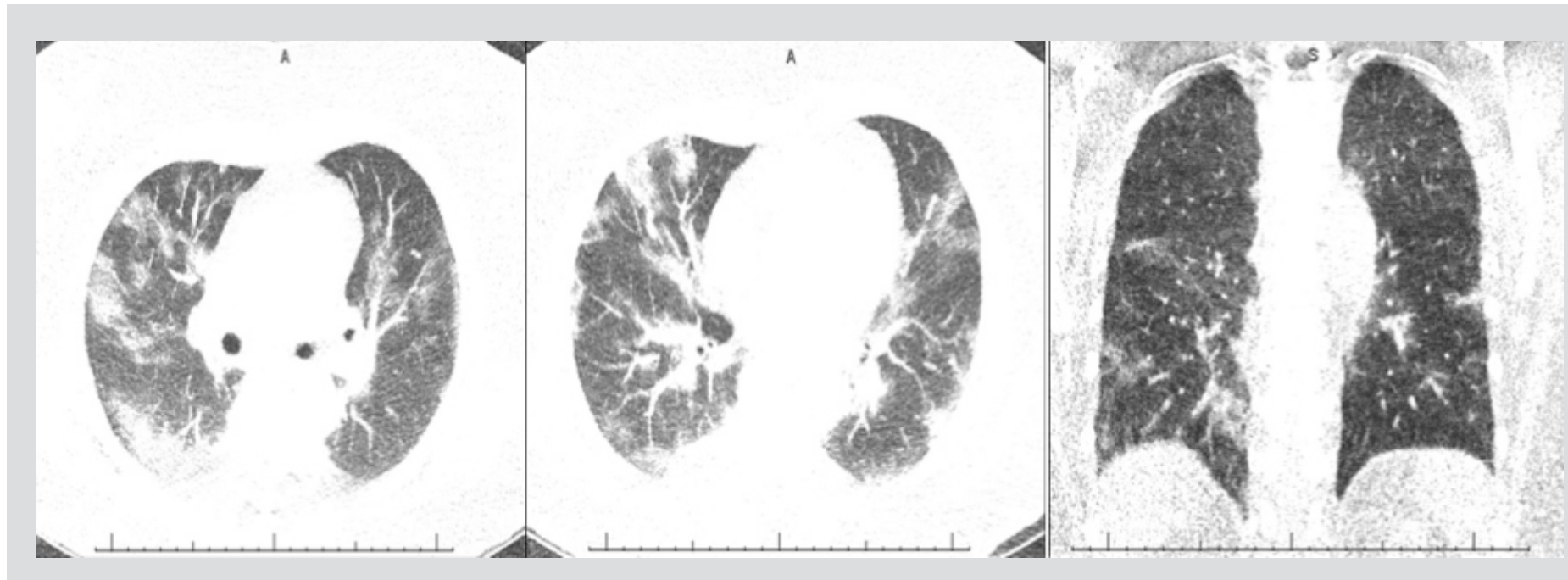

Figure 3. Ultra-low-dose CT images reveal multifocal ground glass opacities with consolidation, suggestive for SARS-CoV-2. Although the radiation dose is comparable to a chest $x$-ray, diagnostic accuracy is better

\section{DISCUSSION}

Our case report underlines the importance of considering the patient's combined clinical characteristics, laboratory results and imaging in diagnosing COVID-19 if the clinical picture is highly suggestive. COVID-19 presentation is heterogeneous, ranging from very mild respiratory symptoms to pneumonia and acute respiratory distress syndrome. There are no specific clinical features or laboratory findings that can reliably distinguish COVID-19 from other viral respiratory infections ${ }^{[4,5]}$. Standard chest CT findings have a sensitivity of up to $97 \%$ compared with RT-PCR assays, but lack specificity ${ }^{[6]}$. The CT manifestations of SARS-CoV-2 pneumonia are similar to those of SARS-CoV-1, which are usually rapidly evolving, multiple and migratory, but the lesions are mainly distributed in the middle and peripheral zones of the lung ${ }^{[7,8]}$. The chest CT abnormalities seen in our patient have also been identified in patients before the detection of viral RNA from upper respiratory specimens ${ }^{[9]}$. Ultra-low-dose chest CT has the advantage over chest radiography of providing radiation doses as low as $0.14-0.5 \mathrm{mSv}$ (compared with $0.1 \mathrm{mSv}$ of PA and LL chest radiography) ${ }^{[10,11]}$ and higher diagnostic sensitivity and specificity ${ }^{[6]}$. In our institution, CT examinations were performed using a multi-detector Somatom Definition Edge CT scanner (Siemens, Erlangen, Germany). The parameters for ultra-low-dose CT were optimized for a patient with a normal BMI between 18.5 and $24.9 \mathrm{~kg} / \mathrm{m}^{2}$. Acquisition parameters were as follows: tube voltage, $80 \mathrm{kVp}$; fix tube current of $20 \mathrm{mAs}$ without automatic exposure control; slice thickness, $2.0 \mathrm{~mm}$; reconstruction interval, $2 \mathrm{~mm}$; with a sharp reconstruction kernel. CT images were obtained with the patient in the supine position at full inspiration and without contrast medium.

False-negative RT-PCR assay results on nasopharyngeal and oral swabs have been described. However, the literature on the sensitivity of nasopharyngeal swab RT-PCR for SARS-CoV-2 is scarce. Few studies have compared the sensitivity of nasopharyngeal and oral swab RTPCR assays with the sensitivity of radiological investigations (in particular chest CT scans) ${ }^{[11-13]}$. Available data indicate the sensitivity of nasopharyngeal and oral swab RT-PCR assays for SARS-CoV-2 ranges between $56 \%$ and $83 \%$, but the methodological quality of published studies on this topic is low ${ }^{[14,15]}$.

Although any estimates of the scale of false-negative results will be inaccurate until observational time spans and numbers of monitored cases are increased, some drawbacks of RT-PCR assays have already been revealed. We have noted the importance of state-of-theart sample collection. The clinical manifestations, laboratory examination results, and chest imaging features of patients with suspected COVID-19 are pivotal for detection and diagnosis. Thus, a combination of several criteria should be established for the diagnosis of SARSCoV-2 infection. If the clinical, laboratory and radiological pictures are highly suggestive of disease, but the results of RT-PCR assays on nasopharyngeal and oral swabs are negative, it might be helpful to perform RT-PCR assays on other specimens, such as urine and stool. Unfortunately, we could not provide these additional analyses for our patient. Given the compelling need to confirm the diagnosis in a patient otherwise considered for diagnostic-driven therapy with tocilizumab or remdesivir, we eventually performed an RT-PCR assay on the BAL. The patient had levels of IL-6 up to $74 \mathrm{pg} / \mathrm{ml}$, almost 20 -fold higher than the normal range (<3.1 pg/ml). However, the patient's clinical status unexpectedly improved and we did not need to administer further treatment. 
Currently, the role of bronchoscopy and BAL in diagnosing SARS-CoV-2 is uncertain. Although bronchoscopy was crucial in our case for the diagnosis of SARS-CoV-2 infection, critical drawbacks remain, particularly the risk of infection and the difficult logistics, as recently reported by the American Association for Bronchology and Interventional Pulmonology (AABIP) ${ }^{[16]}$. Protocols for preventing infection during bronchoscopy should be strictly implemented ${ }^{[17]}$. Thus, the risks and benefits of this procedure should be carefully balanced. We performed bronchoscopy through a laryngeal mask airway under sedation and ventilation (e.g., with a bag valve mask) in order to reduce the risk of infection related to a procedure performed in an alert patient.

In conclusion, we report two RT-PCR assay false-negative results for SARS-CoV-2 infection on nasopharyngeal and oral swabs in a patient clinically and radiologically highly suggestive for COVID-19; the diagnosis was made on RT-PCR assay of BAL. Concerning the imaging modality of choice, ultra-low-dose chest CT, although more expensive, could be a valid option to replace chest x-ray, as it has higher diagnostic accuracy. In clinical practice, if other findings are indicative of infection, it would be unwise to rule out COVID-19 based on the negative results of single or even multiple RT-PCR assays on nasopharyngeal or oral swabs. If there is uncertainty, other biological specimens should be collected and bronchoscopy and BAL could be of help to confirm the diagnosis in eligible patients provided appropriate precautions are taken.

\section{REFERENCES}

1. Gorbalenya AE, Baker SC, Baric RS, de Groot RJ, Drosten C, Gulyaeva AA, et al. The species Severe acute respiratory syndrome-related coronavirus: classifying 2019-nCoV and naming it SARS-CoV-2. Nat Microbiol 2020;5:536-544.

2. Zhu N, Zhang D, Wang W, Li X, Yang B, Song J, et al.; for the China Novel Coronavirus Investigating and Research Team. A novel coronavirus from patients with pneumonia in China, 2019. N Engl J Med 2020;382:727-773.

3. WHO (World Health Organization): Coronavirus disease (COVID-19) Pandemic. Available from: https://www.who.int/emergencies/diseases/novel-coronavirus-2019 (accessed 24 April).

4. Wang D, Hu B, Hu C, Zhu F, Liu X, Zhang J, et al. Clinical characteristics of 138 hospitalized patients with 2019 novel coronavirus-infected pneumonia in Wuhan, China. JAMA 2020;323(11):1061-1069.

5. Huang C, Wang Y, Li X, Ren L, Zhao J, Hu Y, et al. Clinical features of patients infected with 2019 novel coronavirus in Wuhan, China. Lancet 2020;395(10223):497.

6. Ai T, Yang Z, Hou H, Zhan C, Chen C, Lv W, et al. Correlation of chest CT and RT-PCR testing in coronavirus disease 2019 (COVID-19) in China: a report of 1014 cases. Radiology 2020 Feb 26. doi: 10.1148/radiol.2020200642 [Epub ahead of print].

7. Chung M, Bernheim A, Mei X, Zhang N, Huang M, Zeng X, et al. CT imaging features of 2019 novel coronavirus (2019-nCoV). Radiology 2020;295:202-207.

8. Lei J, Li J, Qi X. CT imaging of the 2019 novel coronavirus (2019-nCoV) pneumonia. Radiology 2020;295(1):18.

9. Xie X, Zhong Z, Zhao W, Zheng C, Wang F, Liu J. Chest CT for typical 2019-nCoV pneumonia: relationship to negative RT-PCR testing. Radiology 2020 Feb 12. doi: 10.1148/ radiol.2020200343 [Epub ahead of print]

10. Vilar-Palop J, Vilar J, Hernández-Aguado I, Gonzlez-Ålvarez I, Lumbreras B. Updated effective doses in radiology. J Radiol Prot 2016;36:975-990.

11. Kim Y, Kim YK, Lee BE, Lee SJ, Ryu YJ, Lee JH, et al. Ultra-low-dose CT of the thorax using iterative reconstruction: evaluation of image quality and radiation dose reduction. AJR Am J Roentgenol 2015;204(6):1197-1202.

12. Fang Y, Zhang H, Xie J, Lin M, Ying L, Pang P, et al. Sensitivity of chest CT for Covid-19: comparison to RT-PCR. Radiology 2020 Feb 19. doi: 10.1148/radiol.2020200432 [Epub ahead of print].

13. Dangis A, Gieraerts C, De Bruecker Y, Janssen L, Valgaeren H, Obbels D, et al. Accuracy and reproducibility of low-dose submillisievert chest CT for the diagnosis of COVID-19. Radiol Cardiothor Imaging 2020;2(2).

14. Yang Y, Yang M, Shen C, Wang F, Yuan J, Li J, et al. (2020) Evaluating the accuracy of different respiratory specimens in the laboratory diagnosis and monitoring the viral shedding of 2019-nCoV infections. medRxiv. Available from: https://doi.org/10.1101/2020.02.11.20021493 (accessed 23 April 2020).

15. Wang W, Xu Y, Gao R, Lu R, Han K, Wu G, et al. Detection of SARS-CoV-2 in different types of clinical specimens. JAMA 2020 Mar 11. doi: 10.1001/jama.2020.3786 [Epub ahead of print].

16. Wahidi MM, Lamb C, Murgu S, Musani A, Shojaee S, Sachdeva A, et al. American Association for Bronchology and Interventional Pulmonology (AABIP) Statement on the Use of Bronchoscopy and Respiratory Specimen Collection in Patients with Suspected or Confirmed COVID-19 Infection. 19 March 2020. Available from: https://aabronchology. org/2020/03/12/2020-aabip-statement-on-bronchoscopy-covid-19-infection (accessed 23 April 2020).

17. Group of Interventional Respiratory Medicine, Chinese Thoracic Society. Expert consensus for bronchoscopy during the epidemic of 2019 novel coronavirus infection. Zhonghua Jie He He Hu Xi Za Zhi 2020;43(3):199-202. 